\title{
NECROSE PRIMÁRIA DO CORPO CALOSO (DOENÇA DE MARCHIAFAVA-BIGNAMI)
}

\author{
RELATO DE CINCO CASOS
}

MARIA DE FÁtima FALANGOLA * - ARISTIDES CHETO DE QUEIROZ **

\begin{abstract}
RESUMO - São relatados 5 casos de doença de Marchiafava-Bignami, estudados em seus aspectos anátomo-patológicos, de correlação clínica e de etiopatogenia. O aspecto anátomo-patológico, de desmielinização na parte central do corpo caloso, comprometendo também outras comissuras, é característico da doença. Os autores chamam a atenção para o papel do alcoolismo e da desnutrição na patogênese da lesão e para a predominância, nesta série, de casos do sexo feminino, ocorrência rara entre os casos descritos na literatura.
\end{abstract}

PA L A VRA S - CHA VE: corpo caloso, necrose primária* doença de Marchiafava-Bignami, alcoolismo, desnutrição.

Primary necrosis of the corpus callosum (Marchiafava-Bignaini's disease): report of five case*.

SUMMARY - The clinicopathological study of five autopsied cases of Marchiafava-Bignami disease is reported. The demielination of the central portion of the. corpus callosum is the major characteristics of the disease. In two cases the demielination also involved the anterior and posterior commissures. There is a clear relationship to alcoholism land malnutrition in the cases reported in the present study.

KEY WORDS : corpus callosum, primary necrosis, Marchiafava-Bignami's disease, alcoholism, malnutrition.

Dentre as várias apresentações clínico-patológicas decorrentes da agressão ao sistema nervoso central ( $\mathrm{SNC}$ ) pelo álcool, a doença de Marchiafava-Bignami (DMB ) parece ser a forma menos frequente ${ }^{3 \cdot 5^{9} \cdot 12}$. Do ponto de vista anatomopatológico esta condição se apresenta com quadro de desmielinização ou necrose na parte central do corpo caloso, com extensão variável, podendo atingir também outras comissuras e se estender aos centros semi-ovais 3,5,7,io. Devido ao maior comprometimento do corpo caloso, é denominada também «degeneração central do corpo caloso» 9 ou «desmielinização central do corpo caloso» 3. Sendo condição rara, tem sido descrita mais frequentemente como relato de casos isolados 3-5 ou como pequenos grupos de casos 9. Nessas várias publicações pode-se verificar que suas características anátomo-patológicas são bem definidas, o que permite distinguir de outras condições, particularmente em situações de isquemia do corpo caloso 3,5. um dos aspectos mais interessantes, no estado atual dos conhecimentos sobre a doença, diz respeito à etiopatogenia, particularmente no que se refere à relação com o alcoolismo e desnutrição 3,5,9,io por esta razão o

Trabalho realizado no Serviço de Anatomia Patológica (Setor de Neuropatologia) do Hospital Universitário Prof. Edgard Santos (HUPES) da Universidade Federal da Bahia (UFBa): * Médica do Serviço de Anatomia Patológica-HUPES; ** Chefe do Serviço de Anatomia Patológica-HUPES, Professor Adjunto do Departamento de Anatomia Patológica e Medicina Legal de UFBa.

Dr. Aristides Cheto de Queiroz - Serviço de Anatomia Patológica, Hospital Universitário Prof. Edgard Santos - Vale do Canela - 400ÚO Salvador BA - Brasil. 
estudo, mesmo de casos isolados, deve ser estimulado e dirigido no sentido da relação de causa e efeito desses dois agentes.

Neste estudo são relatados 5 casos de DMB, avaliados em seus aspectos anátomo-patológico, de correlação clínica e de etiopatogenia.

\section{MATERIAL* E MÉTODOS}

São estudados 5 caso» da DMB existentes nos arquivos do Serviço de Anatomia Patológica (Setor de Neuropatologia) do HUPES da UFBa, entre 2804 autópsias realizadas no período de 1970 a 1989. Todos os casos foram submetidos a autópsia completa, com exame macro e microscópico de todos os órgãos. Os encéfalos, no Setor de Neuropatologia, foram estudados após fixação em formol a 10\%, em. suspensão, por período nunca inferior a 15 dias. As secções obtidas para estudos histopatológicos, pela técnica de inclusão em parafina, foram coradas pela hematoxilina eosina, pela hematoxilina férrica de Lopes para demonstração da bainha de mielina e pela coloração de Glees-Marsland para demonstração do axônio Os prontuários clínicos foram consultados para obtenção de dados de correlação clínico-patológica.

\section{RESULTADOS}

Os principais dados anátomo-patológicos dos casos estão expressos na Tabela 1, na qual se mostra que a lesão atinge de maneira acentuada o corpo caloso. Há envolvimento de toda a extensão desta estrutura em 4 casos e considerável redução da espessura (Fig. 1). O comprometimento de outras estruturas comissurais foi observado em 2 casos. Lesão associada frequente neste grupo foi a presença de atrofia cerebral, representada por alargamento dos sulcos e estreitamento dos giros, ao lado de dilatação do sistema ventricular de grau moderado. No estudo histológico, há nítida degeneração de mielina em graus variáveis ao nível do corpo caloso, com preservação dos axônios (Figs. 2 e 4) \& com reação glial e vascular (Fig. 3).

Alguns dados importantes da correlação clínica podem ser observados na Tabela 2. Chama atenção a faixa etária, com ocorrência em indivíduos acima dos 40 anos e com predominância do sexo feminino. É importante chamar atenção pana a correlação a alcoolismo em 4 casos e ao quadro nutricional, sempre precário, em todos os casos. Em nenhum dos casos havia manifestação neurológica que pudesse ser correlacionada à apresentação anatomopatológica da doença.

Tabela 1. Doença de Marchilafava-Bignami: dados anátomo-patológicos do encéfalo de 5 casos necropsiados.

\begin{tabular}{|c|c|c|c|c|c|}
\hline Caso & $\begin{array}{l}\text { Peso do } \\
\text { encéfalo }\end{array}$ & $\begin{array}{l}\text { Extensão lesão } \\
\text { corpo caloso }\end{array}$ & $\begin{array}{l}\text { Espessura } \\
\text { corpo caloso }\end{array}$ & $\begin{array}{c}\text { Lesão outras } \\
\text { comissuras }\end{array}$ & Outras lesões \\
\hline 1 & $1300 \mathrm{~g}$ & toda & $0,3 \mathrm{~cm}$ & sem referência & $\begin{array}{l}\text { atrofia e } \\
\text { aterosclerose }\end{array}$ \\
\hline 2 & $1100 \mathrm{~g}$ & toda & $0,2 \mathrm{~cm}$ & $\begin{array}{l}\text { anterior e } \\
\text { posterior }\end{array}$ & ausentes \\
\hline 3 & $1200 \mathrm{~g}$ & $\begin{array}{l}\text { frontal e } \\
\text { parietal }\end{array}$ & $0,3 \mathrm{~cm}$ & sem referência & atrofia \\
\hline 4 & $900 \mathrm{~g}$ & toda & $0,3 \mathrm{~cm}$ & sem alterações & atrofia \\
\hline 5 & $1150 \mathrm{~g}$ & toda & $0,3 \mathrm{~cm}$ & posterior & atrofia \\
\hline
\end{tabular}




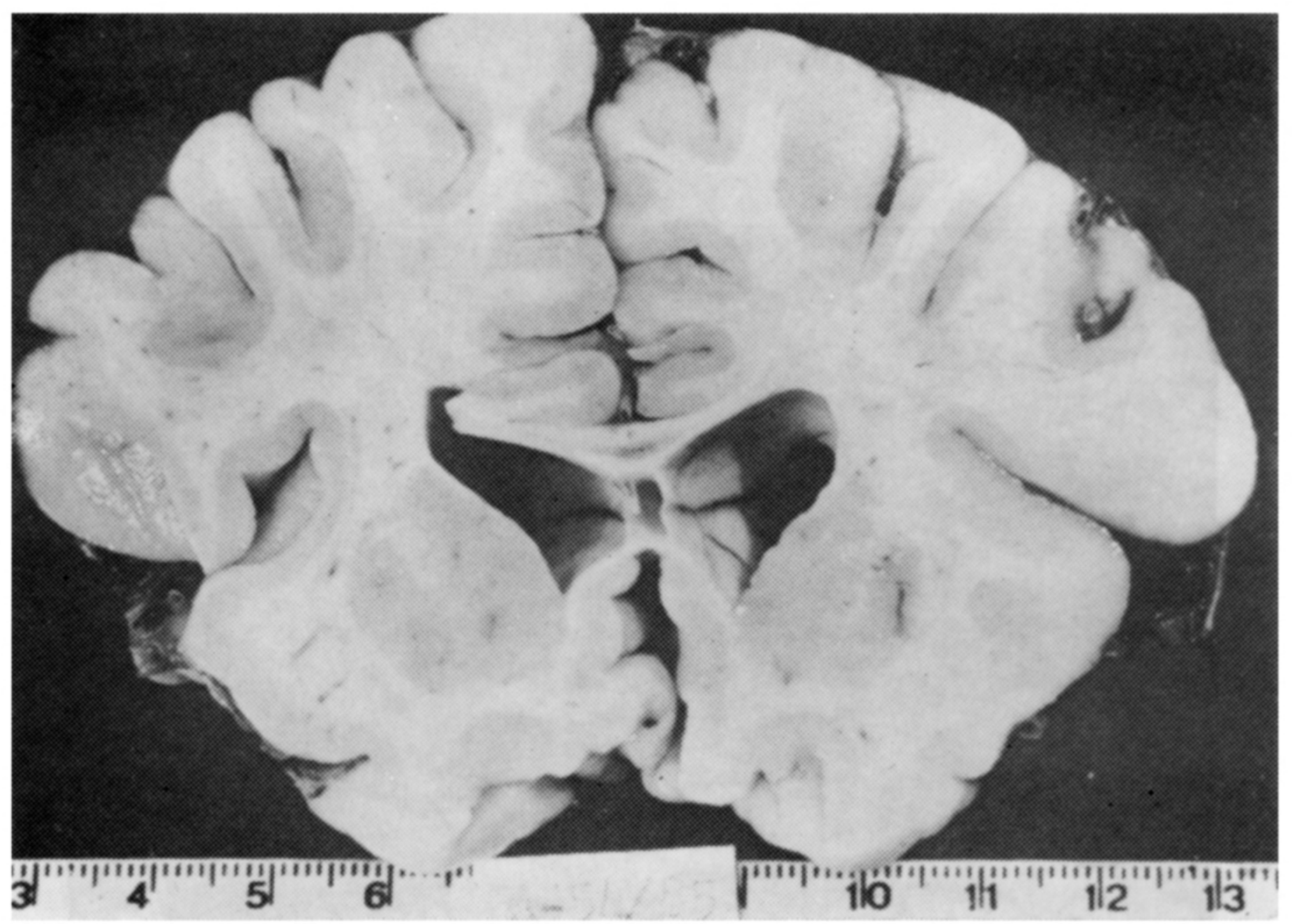

Fig. 1. Corpo caloso na sua porção mais anterior com redução da espessura e evidente aspécto degenerativo da parte central (aspécto em sanduiche).

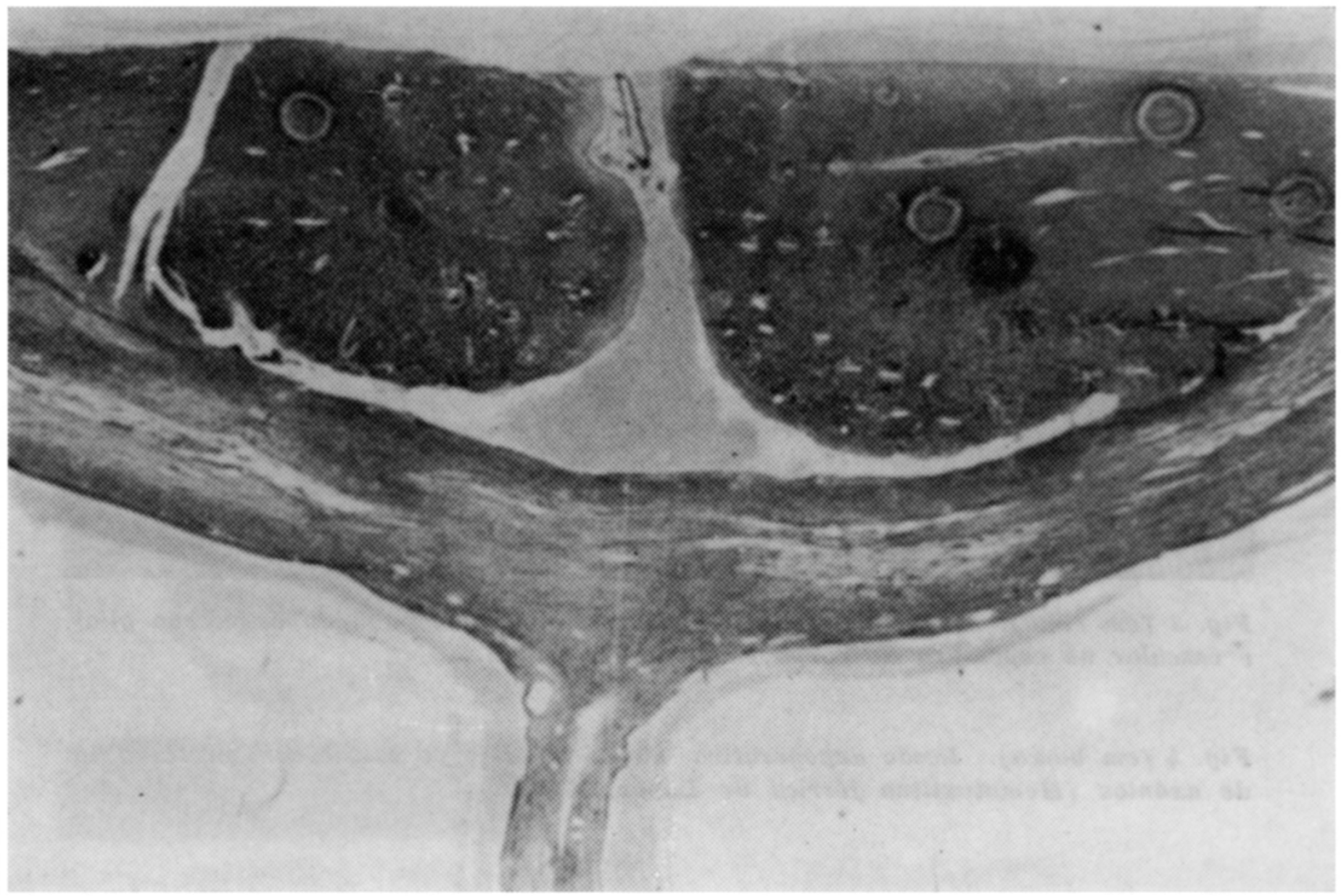

Fig. 2. Seç̧̃ão histológica do corpo caloso mostrando degenração da mielina na sua espessura $(H E, \times 10)$. 

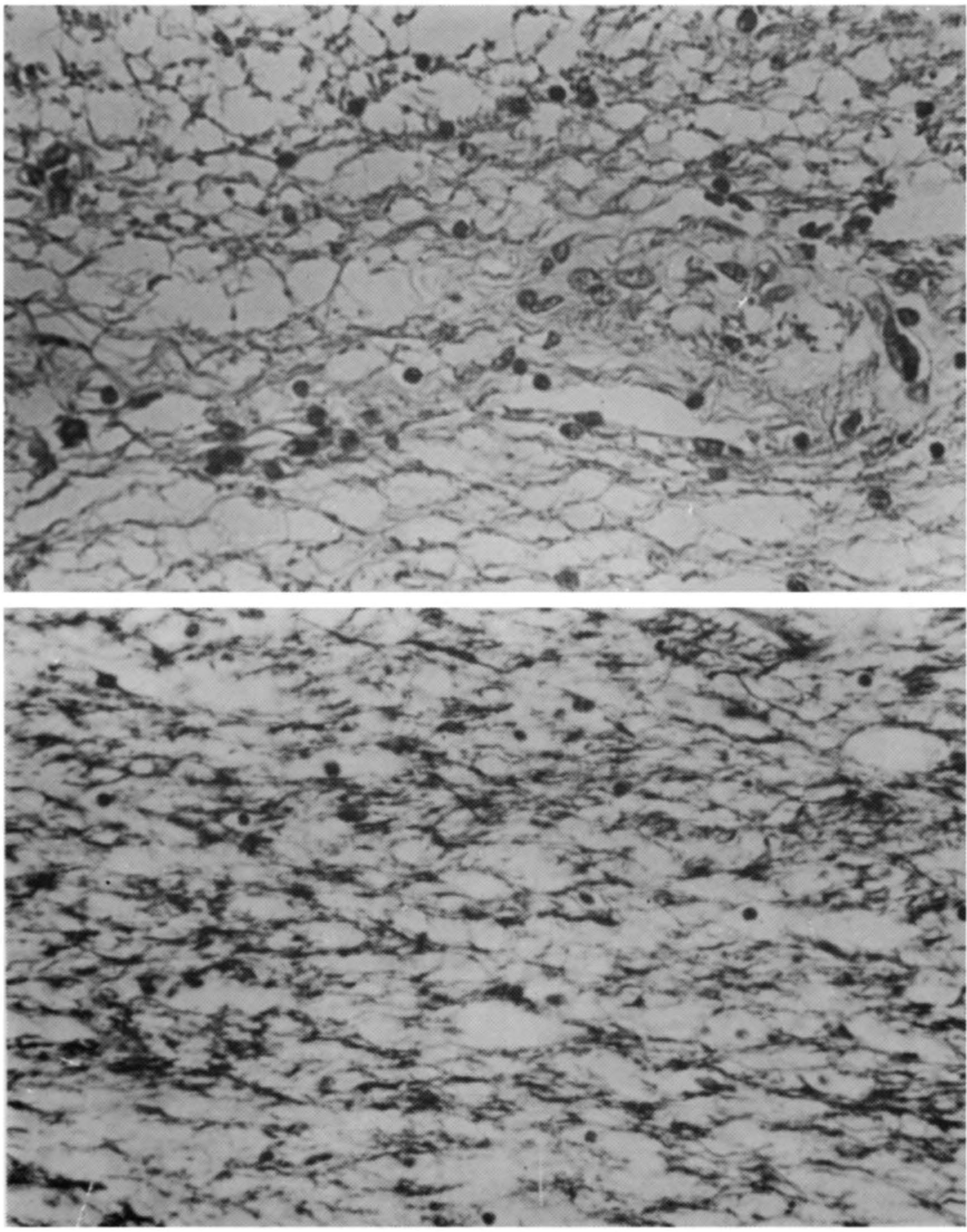

Fig. 3 (em cima). Aspecto da degeneração mielinica acompanhadia de reação glial e vascular na espessura do corpo caloso $(H E, \times 400)$.

Fig. 4 (em baixo). Lesão degenerativa mielinica do corpo caloso com prcservação de axônios (Hematoxilina ferrica de Lopes, $\times 400)$. 
rabela 2. Doença de Marchiafava-Bignami: dados clínico-patológicos de 5 casos necropsiados.

\begin{tabular}{|c|c|c|c|c|c|c|}
\hline Caso & $\begin{array}{l}\text { Idade } \\
\text { (anos) }\end{array}$ & Sexo & Doença básica & Causa mortis & $\begin{array}{c}\text { Estado } \\
\text { nutricional }\end{array}$ & Alcoolismo \\
\hline 1 & 58 & Fem & $\begin{array}{l}\text { Hodgkin } \\
\text { disseminado }\end{array}$ & $\begin{array}{l}\text { Hodgkin } \\
\text { disseminado }\end{array}$ & precário & sem relato \\
\hline 2 & 41 & Fem & $\begin{array}{l}\text { Pseudocisto } \\
\text { pâncreas }\end{array}$ & $\begin{array}{l}\text { Hemorragia } \\
\text { digestiva }\end{array}$ & precário & moderado \\
\hline 3 & 54 & Masc & $\begin{array}{l}\text { Histoplasmose } \\
\text { disseminada }\end{array}$ & $\begin{array}{l}\text { Insuficiência } \\
\text { supra-renais }\end{array}$ & precário & moderado \\
\hline 4 & 76 & Fem & $\begin{array}{l}\text { Cardiopatia } \\
\text { hipertensiva }\end{array}$ & $\begin{array}{l}\text { Insuficiência } \\
\text { cardiaca } \\
\text { congestiva }\end{array}$ & precário & intenso \\
\hline 5 & 71 & Masc & $\begin{array}{l}\text { Ca. de pulmão } \\
\text { Diab. mellitus }\end{array}$ & $\begin{array}{l}\text { Insuficiência } \\
\text { respiratória }\end{array}$ & precário & intenso \\
\hline
\end{tabular}

\section{COMENTÁRIOS}

Os aspectos anátomo patológicos dos 5 casos são característicos e permitem, de maneira bem definida, o diagnóstico da doença de Marchiafava-Bignami, seguindo-se os critérios estabelecidos em vários estudos 3,5,6,9. É interessante frizar que, mesmo sendo denominada degeneração central do corpo caloso, a lesão nào é exclusiva desta estrutura, pois o processo de desmielinização compromete também outras comissuras e até mesmo a substância branca dos centros semi-ovais $3,8,12$.

Estes nossos casos mostram uma particularidade no que se refere ao sexo. Diferentemente do que está mencionado na maioria dos trabalhos da literatura, em nosso material houve predominância do sexo feminino. Durante muito tempo pensou-se que a doença afetasse exclusivamente pessoas do sexo masculino, até que Schwob e col." publicaram um bem documentado caso do sexo feminino. Posteriormente, outros poucos casos foram descritos?. Não há, entretanto, na literatura qualquer dado que justifique claramente essa predominância.

Aspecto importante no estudo desta condição é a relação etiopatogênica com o alcoolismo, mencionado desde as primeiras descrições da doença, quando da sua associação à ingestão de vinhos, entre italianos 3,9. Em nosso material, esta relação com o alcoolismo é bem nítida e fortalece a importância deste fator quanto à lesão desmielinizante do corpo caloso.

A atrofia cerebral, que foi vista em 4 casos, não mantém relação de causa e efeito com alterações vasculares cerebrais. É possível que esta alteração possa estar relacionada ao alcoolismo crônico ${ }^{13}$, uma das lesões dentre as várias descritas em associação com esta droga. A associação da DMB com outras lesões descritas no alcoolismo já é bem conhecida, inclusive à mielinólise pontina ${ }^{2}$. Tais dados só fortalecem a importância do papel do alcoolismo no desenvolvimento da doença.

A descrição desta doença em pessoas reconhecidamente não alcoólatras', embora rara, pode reforçar a hipótese de que outros fatores possam estar relacionados, como a desnutrição ${ }^{\prime}{ }^{13}$ e agentes tóxicos 3,5 . Sato e col. ${ }^{1}$ (C) descreveram o caso de um paciente japonês bebedor de sakê e refutam a hipótese de uma ação direta do álcool, pois este tipo de bebida contém teor alcoólico baixo (14\%), e sugerem que a lesão é provavelmente secundaria a deficiência nutricional relacionada com vitaminas do grupo B. Para Ma Kuo-Chun e Chen Kuan-Jung ${ }^{6}$, que descreveram o caso de um paciente chinês não etilista e em bom estado nutricional, há evidências em relação a distúrbio metabólico severo. Além disso, envolvimento seletivo do corpo caloso pode ser induzido experimentalmente por intoxicação com cianetos. 
Dessa maneira, ainda hoje, muitos são os fatores que podem estar relacionados à patogênese dessa lesão e neste estudo é chamada a atenção para pelo menos dois deles, o alcoolismo e a desnutrição, ressaltando a necessidade de se dar ênfase ao papel que, isolados ou em conjunto, possam desempenhar.

\section{REFERÊNCIAS}

1. Behmer OA, Tolosa EMC, Freitas AG Neto. Manual de Técnicas para Histología Normal e Patológica, São Paulo: EDART, 1976.

2. Ghatak NR, Hadfield MG, Rosenblum WI. Association of central pontine myelinolysis and Marchiafava-Bignami disease. Neurology 1978, 28:1295-1298.

3. Ironside R, Bosanquet FD, McMengmy WH. Central demyelination of the corpus callosum (Marchiafava-Bignami disease) with report of a second case in Great Britain. Brain 1961, 84:212-230.

4. Ishizaki $\mathrm{T}$, Chitanondh $\mathrm{H}$, Laksanavicharn $\mathrm{W}$. Marchiafava-Bignami's disease: report of the first case in Asia. Acta Neuropathol 1970, 16:187-193.

5. Koepeen AH, Barron KD. Marchiafava-Bignami disease. Neurology 1978, 28:290-294.

6. Kuo-Chun Ma, Kuan-Jung C. Primary necrosis of corpus callosum with dystrophic astrogliosis and Rosenthai-like fiber formation: the first Chinese case of Màrchiafava-Bignami's disease. Acta Neuropathol 1983, 60:283-290.

7. McLardy Turner MBE. A case of Marchiafava's disease (primary degeneration of the corpus callosum). Proc. R Soc Med 1951, 44:685-686.

8. McMenemey WH, Smith WT. The central nervous system. Vol 5. In Symmers W St Clair (ed): Systemic Pathology, Vol 5. Edinburgh: Churchill Livingstone, 1979.

9. Merrit $\mathrm{HH}$, Weisman AD. Primary degeneration of corpus callosum (Marchiafava-Bignami's disease). J Neuropathol Exp Neurol 1945, 4:155-163.

10. Sato Y, Tabira T, Tateishi J. Marchiafava-Bignami disease, striatal degeneration and other neurological complications of chronic alcoholism in a Japanese. Acta Neuropathol 1981, 53:15-20.

11. Schwob RA, Gruner J, Foucquier E. Sur trois cas de syndrome confusionnel avec contrature cher des ethniques: le problème des encephalopathies carentielles. Rev Neurol (Paris) 1953, 88:174-190

12. Testa U. Lesions of corpus callosum in experimental alcoholism. Riv Sper Freniat 1929, 52:559-574.

13. Torvic A, Lindboe CF, Rogde C. Brain lesions in alcoholics: a neuropathological study with clinical correlations. J Neurol Sci 1982, 56:233-248. 